\title{
New Imaging Findings of Incomplete Partition Type III Inner Ear Malformation and Literature Review
}

\author{
(D) R. Hong, (D) Q. Du, and (D) Y. Pan
}

\begin{abstract}
SUMMARY: Incomplete partition type III, also referred to as X-linked deafness, is a rare genetic inner ear malformation. Its characteristic CT findings, including bulbous dilation of the internal auditory canal and absence of the modiolus with the interscalar septa present, have been well-recognized. In this series of 19 cases, we report the abnormalities of the vestibule and semicircular canals and provide a comprehensive description of their CT and MR imaging findings. The inner ear malformations in incomplete partition type III were bilateral and basically symmetric, with involvement of the internal auditory canal, nerve canals in the fundus, cochlea, vestibule, semicircular canals, vestibular aqueduct, otic capsule, round window, oval window, and stapes. An irregular vestibule with a cystic appearance is also a distinctive imaging feature, which could be seen in about $90 \%$ of our patients, with a cystic appearance of the semicircular canals present in nearly half of the cases.
\end{abstract}

ABBREVIATIONS: $\mathrm{CN}=$ cochlear nerve; DFNX2 = X-linked deafness type 2; IAC = internal auditory canal; IP-III = incomplete partition type III; SCC = semicircular canal; $\mathrm{VA}=$ vestibular aqueduct; $\mathrm{VN}=$ vestibular nerve

n 1971, Nance et $\mathrm{al}^{1}$ described X-linked mixed deafness with congenital fixation of the stapedial footplate and perilymphatic gusher. Based on the classification of sex-linked loci and genes implicated in nonsyndromic deafness, this type of hearing loss has been classified as X-linked deafness type 2 (DFNX2), and the responsible gene is POU class 3 homeobox 4 (POU3F4). ${ }^{2}$ Its distinctive CT features were first described by Phelps et $\mathrm{al}^{3}$ in 1991, and it was named incomplete partition type III (IP-III) by Sennaroglu et $\mathrm{al}^{4}$ in 2006.

The characteristic CT findings of the cochlea and internal auditory canal (IAC) in IP-III have been described in detail, including absence of the modiolus with interscalar septa present and a bulbous dilated IAC at the lateral end. ${ }^{3-7}$ However, there are fewer descriptions of abnormal vestibules and semicircular canals (SCC). By analyzing a case series of 19 patients with IP-III, we aimed to present the newly described imaging findings of the vestibular and

Received December 28, 2019; accepted after revision April 4, 2020.

From the Departments of Radiology (R.H., Y.P.) and Otolaryngology-Head and Neck Surgery (Q.D.), Eye and ENT Hospital of Shanghai Medical School, Fudan University, Shanghai, China

Please address correspondence to Yucheng Pan, MD, Department of Radiology, Eye and ENT Hospital of Shanghai Medical School, Fudan University, 83 Fenyang Rd, Shanghai 200031, China; e-mail: panyuchengg@sina.com

Indicates article with supplemental on-line tables.

Indicates article with supplemental on-line photo.

http://dx.doi.org/10.3174/ajnr.A6576
SCC abnormalities in IP-III and provide a comprehensive description of its CT and MR imaging features with a literature review.

\section{MATERIALS AND METHODS}

This retrospective case series was performed with the approval of the institutional review board and exemption from informed consent. Imaging records of 2075 patients with inner ear malformation (based on the classification of Sennaroğlu and Bajin ${ }^{8}$ ) between August 2014 and December 2018 were reviewed, and 19 patients ( 18 males and 1 female, 6 months to 47 years of age) with the IP-III anomaly were finally included. Genetic analyses were not performed.

All 19 patients underwent CT examinations of the temporal bone, and 14 patients underwent MR imaging examinations. The CT scans were performed with a 16- or a 128-section multidetector CT scanner (Somatom Sensation 16 or Definition Edge; Siemens). Image acquisition and reconstruction parameters were as follows: helical acquisition, $120 \mathrm{kV}, 240$ effective mAs, 0.75 pitch, 0.75 - or 0.6- $\mathrm{mm}$ thickness, 0.75 - or 0.6-mm collimation, 220-mm FOV, and B70 reconstruction kernels. Multiplanar reformatted images were processed on a separate workstation (Carestream RIS 3.1; https:/www.carestream.com/en/us/ris-software). The temporal bone MR images were obtained with a 3T MR imaging unit (Magnetom Verio; Siemens) using matched 12-channel phased array coils. The MR imaging protocol included an axial sampling perfection with application-optimized contrasts by using different flip angle evolution (SPACE; Siemens) scan plane (3D-SPACE) 
sequence with 0.5 -mm thickness, oblique sagittal 3D-CISS images perpendicular to IAC with $1-\mathrm{mm}$ thickness, and routine an axial and coronal T1WI and T2WI. Two experienced neuroradiologists and 1 otologist evaluated these CT and MR imaging findings.

The cochlear dimensions, including basal turn length and lumen diameter and upper turn width and height, were measured according to the method of Purcell et $\mathrm{al}^{9}$ (On-line Figure). Fifteen cases without inner ear malformation were selected as a healthy control group. IP-III and healthy groups were compared using a $t$ test.

\section{RESULTS}

The prevalence of IP-III among cases of total inner ear anomaly was calculated to be $0.9 \%$ (19/2075). The inner ear malformations in IP-III were bilateral and basically symmetric with involvement of the IAC, nerve canals in the IAC fundus, cochlea, vestibule, SCC, vestibular aqueduct (VA), otic capsule, oval and round windows, and stapes. The imaging findings are shown in the Table.

All 19 patients had characteristic cochlear and IAC appearances as follows: bulbous dilation of the lateral end of the IAC, absence of the bony modiolus with the interscalar septa present, hypoplasia at the cochlear base (or enlargement of the cochlear nerve [CN] canal), and thinning of the otic capsule (Fig 1A,-B). The outline of the cochlea was similar to normal, but the dimensions of the cochlea were slightly smaller than normal (On-line Tables 1 and 2), including basal turn length (7.91-mm versus $8.66-\mathrm{mm}, P=.000$ ),

\section{Imaging findings of the patients}

\begin{tabular}{lcl}
\hline \multicolumn{1}{c}{ Imaging Findings } & Cases & \multicolumn{1}{c}{ First Reported, Year } \\
\hline Dilated internal auditory canal & 19 & Phelps et al, ${ }^{3} 1991$ \\
Hypoplasia at the cochlear base & 19 & Phelps et al, ${ }^{3} 1991$ \\
Absence of the bony modiolus & 19 & Talbot and Wilson, ${ }^{5} 1994$ \\
Thin otic capsule & 19 & Sennaroglu, ${ }^{13} 2016$ \\
Enlarged superior vestibular nerve canal & 16 & Saylisoy et al, ${ }^{6} 2014$ \\
Enlarged labyrinthine facial nerve canal & 11 & Phelps et al, ${ }^{3} 1991$ \\
Enlarged singular nerve canal & 8 & Talbot and Wilson, ${ }^{5} 1994$ \\
Vestibule with cystic appearance & 17 & Gong et al, ${ }^{7} 2014$ \\
Semicircular canal with cystic appearance & 9 & Anderson et al, ${ }^{14} 2020$ \\
Dilated vestibular aqueduct & 7 & Talbot and Wilson, ${ }^{5} 1994$ \\
Dysplasia of oval window & 12 & Saylisoy et al, ${ }^{6} 2014$ \\
Dysplasia of round window & 11 & Saylisoy et al, ${ }^{6} 2014$ \\
Abnormal stapes & 5 & Saylisoy et al, ${ }^{6} 2014$ \\
\hline
\end{tabular}

basal turn lumen diameter (1.78-mm versus $1.93-\mathrm{mm}, P=.004)$, and upper turn width $(4.59-\mathrm{mm}$ versus $5.87-\mathrm{mm}, P=.000)$. However, the upper turn height showed no significant difference between the IP-III and healthy groups $(3.45-\mathrm{mm}$ versus $3.51-\mathrm{mm}$, $P=.258)$. The $\mathrm{CN}$ and vestibular nerve (VN) were visible in all 14 patients who underwent MR imaging, and there was a hypointense spiral structure extending from the fundus of the IAC to the cochlea in 13 patients (Fig $1 C,-D$ ).

In addition to the markedly widened $\mathrm{CN}$ canal, the superior $\mathrm{VN}$ canal was enlarged in 16 patients (Fig $2 A$ ), and the canals of the small branches of the superior $\mathrm{VN}$ from the ampulla of the lateral SCC and the utricle were also visible. The labyrinthine facial nerve canal was slightly enlarged in 11 patients (Fig 2A), and the singular nerve canal, in 14 ears of 8 patients (Fig $2 B$ ). The inferior VN canal showed no enlargement. On MR imaging, 14 patients with enlarged superior $\mathrm{VN}$ canals and 6 patients with enlarged labyrinthine facial nerve canals demonstrated fluid signal (Fig 2C). Twelve patients showed fluid signal in the singular nerve canal (Fig 2D), and 6 of them did not show enlargement of the singular nerve canal.

An irregularly shaped vestibule with small cystic bulges in the margin was observed in 17 patients (Fig 3). The small sacs were usually located in the superior margin, between the feet of the superior SCC. A multicystic appearance of SCC was also seen in 9 patients (Fig 4), with a widened lumen of the SCC in 1 patient. Enlargement of the VA at the part close to the vestibule was observed in 13 ears of 7 patients, with a cystic appearance present in 8 ears and a normal VA orifice (Fig 2A). No patient showed an enlarged endolymphatic sac on MR imaging.

The oval window was dysplastic in 12 patients, and the round window, in 11 patients, including 1 patient with bilateral oval and round window atresia (Fig 5). A thickened stapedial footplate was observed in 5 patients, with malformed stapes in 1 patient.

\section{DISCUSSION}

$\mathrm{X}$-linked nonsyndromic deafness has been estimated to contribute only $1 \%-$
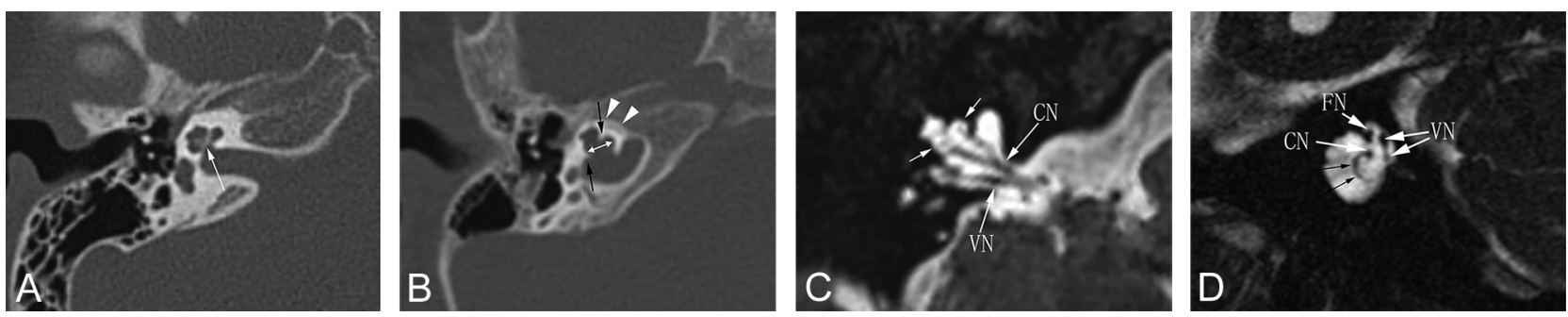

FIG 1. A, Axial CT image of a normal inner ear shows the normal shape of the internal auditory canal, cochlea, modiolus (arrow), and otic capsule. CT and MR images of a 7-month-old male patient's right ear. $B$, Axial CT image shows a bulbous dilated internal auditory canal and the absence of the modiolus with the interscalar septa present (black arrows) and thinning of the otic capsule (arrowheads), with mild pericochlear hypodensity. The middle turn width of the cochlea is shorter than normal (white arrow). C, Axial SPACE image shows the CN, VN, and the hypointense structure in the middle turn of the cochlea (short arrows). The branches of the CN are also shown. $D$, Oblique sagittal CISS image perpendicular to the internal auditory canal shows the facial nerve (FN), CN, and VN. There is a spiral structure (black arrows) extending from the fundus of internal auditory canal to the cochlea, which is connected to the cochlear nerve. 

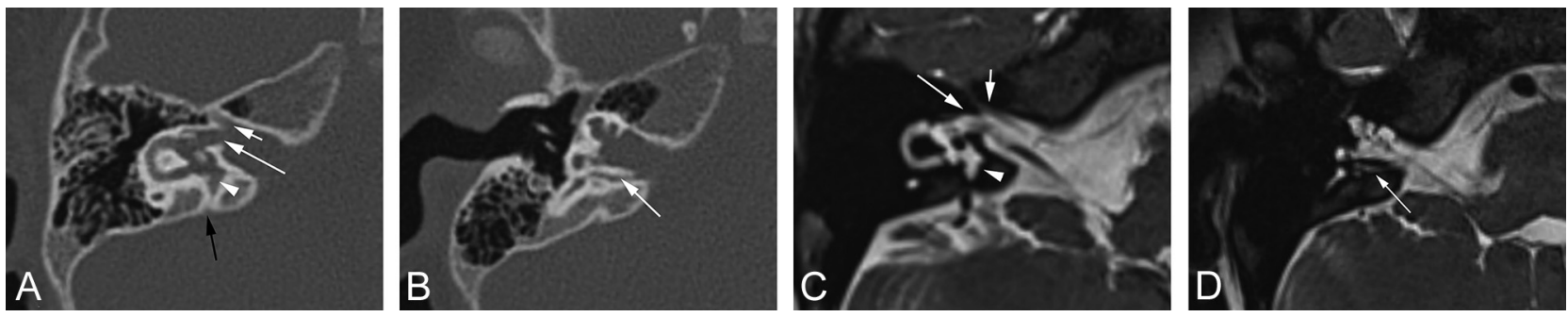

FIG 2. CT and MR images of a 3-year-old male patient's right ear. A, Axial CT image shows an enlarged labyrinthine facial nerve canal (short white arrow), enlarged superior vestibular nerve canal (long white arrow), enlargement of the vestibular aqueduct with a cystic appearance at the part close to the vestibule (arrowhead), and the normal vestibular aqueduct orifice (black arrow). B, Axial CT image shows the enlarged singular nerve canal (arrow) and absence of the modiolus. C, Axial SPACE image shows fluid signal in the enlarged labyrinthine facial nerve canal (short white arrow), superior vestibular nerve canal (long white arrow), and the vestibular aqueduct (arrowhead). D, Axial SPACE image shows fluid signal in the singular nerve canal (arrow).
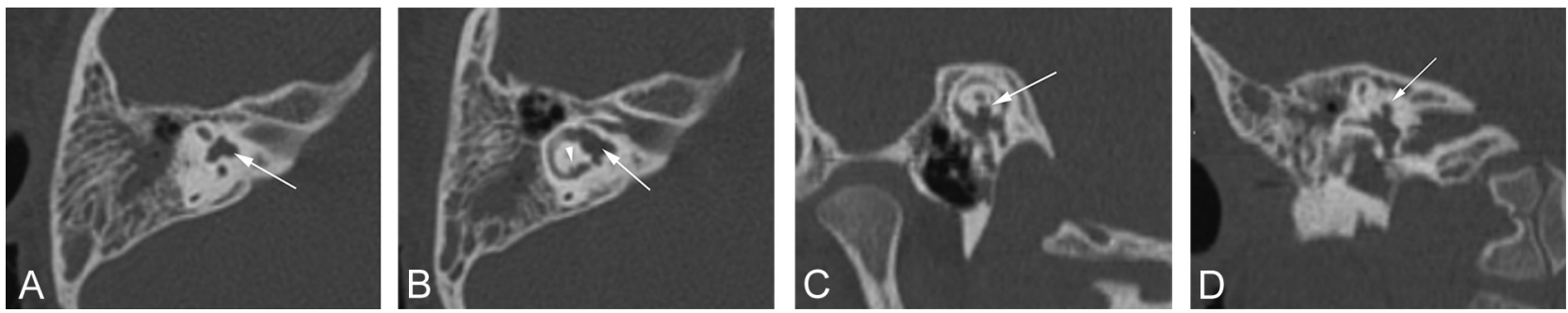

FIG 3. $C T$ images of a 4-year-old male patient's right ear. $A$, Axial $C T$ image shows a cystic bulge between the superior semicircular canal feet (arrow). B, Axial CT image shows the irregularly shaped vestibule (arrow) and small cystic bulges at the lateral semicircular canal (arrowhead). C and $D, M P R C T$ image of the superior semicircular canal and coronal CT image show the cystic bulge of the vestibule protruding upward (arrow).
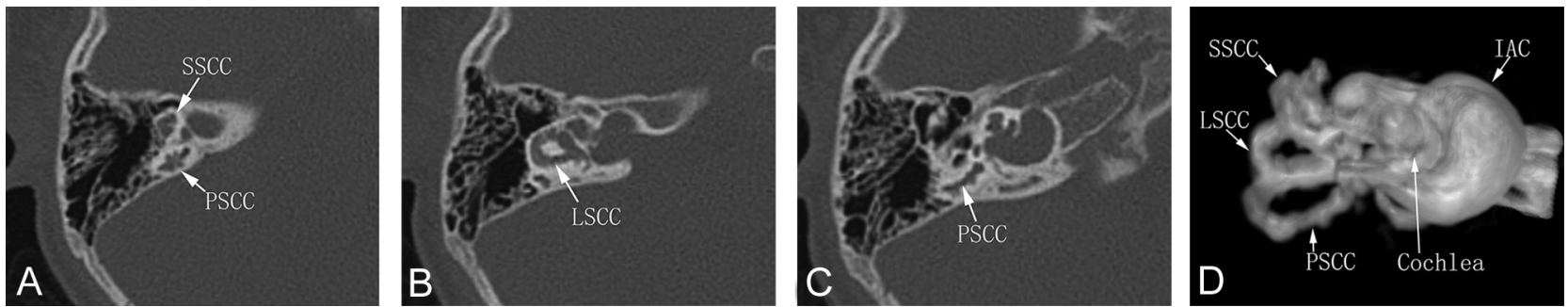

FIG 4. A-C, Axial CT images of a 3-year-old male patient's right ear show multicystic appearance at the superior semicircular canal (SSCC), posterior semicircular canal (PSCC), and lateral semicircular canal (LSCC). Bulbous dilation of the IAC and absence of modiolus are also demonstrated. D, A 3D MR hydrography image shows the cystic appearance of the semicircular canals and dilation of the IAC.

$5 \%$ of genetic nonsyndromic deafness. ${ }^{2}$ DFNX2 is the most common form of X-linked nonsyndromic deafness, which accounts for approximately $50 \%$ of all families with X-linked nonsyndromic deafness. ${ }^{2}$ Most interestingly, DFNX2 is the only form of X-linked deafness to show anatomic anomalies on CT of the temporal bone. ${ }^{2}$ Sennaroglu et $\mathrm{al}^{4}$ considered these anomalies as a type between incomplete partition type I (cystic cochleovestibular malformation) and incomplete partition type II (classic Mondini deformity) and called it IP-III. The term IP-III could help radiologists better understand this rare and characteristic type of deformity in the comprehensive classification of inner ear malformations. Choi et $\mathrm{al}^{10}$ identified IP-III in 10 (4.8\%) of 206 patients with inner ear abnormalities in their research. Sennaroğlu and Bajin $^{8}$ reported that IP-III constituted $2 \%$ of inner ear malformations in their data base. In our case series, it only accounted for $0.9 \%$. IP-III mainly occurs in males, but female carriers may have milder forms of some characteristic findings. ${ }^{6,11,12}$

Phelps et $\mathrm{al}^{3}$ first reported the following characteristic CT findings: bulbous IACs, incomplete separation of the basal turn of the cochleae from the fundi of the IAC, and wide first and second parts of the intratemporal facial nerve canals. Talbot and Wilson ${ }^{5}$ later added absence of the bony modiolus, an abnormal VA, and a dilated singular nerve canal. Sennaroglu ${ }^{13}$ noted that the otic capsule around the membranous labyrinth was thinner than normal. Saylisoy et $\mathrm{al}^{6}$ observed an enlarged VA with a cystic appearance and middle ear anomalies, including dysplasia at the oval and/or round window and stapes abnormalities such as a thickened footplate and single crus. Gong et $\mathrm{al}^{7}$ reported the stapes abnormalities, 

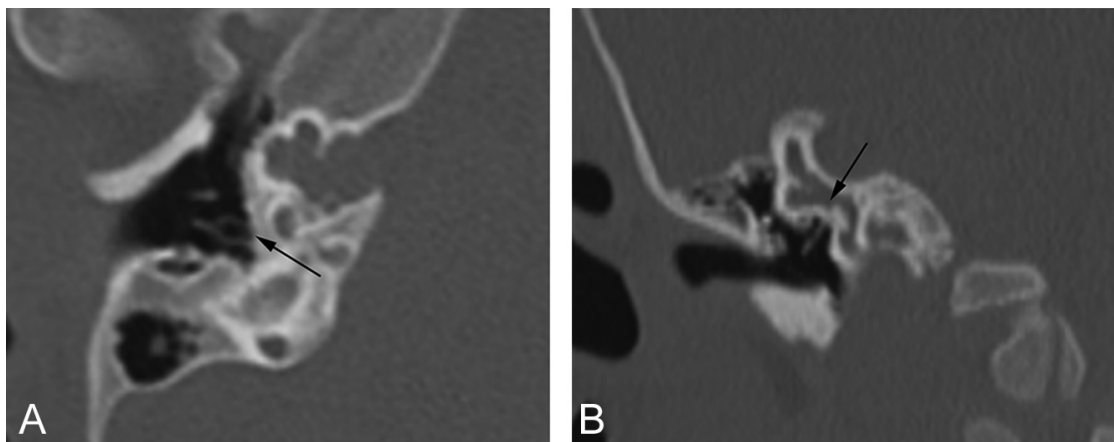

FIG 5. CT images of a 19-month-old male patient's right ear. A, Axial CT image shows thickness of the stapedial footplate and atresia of the oval window (arrow) and absence of the modiolus. $B$, Coronal CT image shows atresia of the oval window (arrow).

enlarged superior VN canal, and the cystic bulge in the superior vestibular margin. Anderson et al ${ }^{14}$ described the cystic changes in the SCC and vestibule, and they also reported a potential association between IP-III and hypothalamic hamartoma.

This case series demonstrates that the entire bony labyrinth, otic capsule, and IAC are all involved in IP-III. A dilated IAC at the lateral end and absence of modiolus with interscalar septa present are the most typical imaging findings. The size of the cochlea is slightly smaller, especially in the middle turn width, but the upper turn height is within the normal range. Although the cochlear cavity appears to be empty on CT, a hypointense spiral structure can be observed on MR imaging. This structure probably represents the membranous labyrinth, which extends into the dilated IAC due to the incomplete separation of the cochlear base from the IAC, and correspondingly, the branches of the $\mathrm{CN}$ can also be observed in the IAC. The enlarged superior VN canal has some noteworthy details as well. Because the superior VN has several branches from the ampullae of superior and lateral SCC and the utricle, the enlarged bony canals of some of these small branches can also be seen in some cases. An irregular vestibule with a cystic appearance is also a very common imaging finding, which could be seen in about $90 \%$ of our cases, and a cystic appearance of the SCC was present in nearly half of our patients. A slightly enlarged VA with a cystic appearance can also be observed in some cases. These cystic bulges show the same signal as the inner ear fluid on MR imaging. Dysplasia of the oval and round windows is also common, and the stapes footplate is sometimes involved.

DFNX2 hearing loss is due to loss of function of the POU3F4 protein. ${ }^{2}$ The POU3F4 gene is expressed in early embryos in the otic capsule, which is involved in mesenchymal-mesenchymal signaling for the development of the inner ear. ${ }^{2}$ During the inner ear development of the mouse, the Brn4/Pou3f4 gene product is initially detected in the ventral aspect of the otic capsule and later throughout the otic capsule in the mesenchyme of both the cochlear and vestibular aspects. ${ }^{15}$ The subcellular localization of the Brn4/Pou3f4 gene product also changes during the differentiation of the otic capsule. It remains nuclear in those regions of the otic capsule that eventually give rise to the mature bony labyrinth. However, the subcellular localization of the product shifts from strictly nuclear to perinuclear in those regions of the otic capsule that will cavitate to form acellular regions in the temporal bone, such as the scala tympani, scala vestibuli, and the internal auditory meatus. ${ }^{15}$ Brn4/Pou3f4-deficient mice also show very similar anomalies to those in human DFNX2. ${ }^{16}$ Most of the phenotypic features of these mutant animals result from the reduction or thinning of the bony compartment of the inner ear, including malformations of the stapes, cochlea, IAC, and superior SCC..$^{15}$

The abnormal development of the otic capsule should be the key factor in these malformations in IP-III. It may lead to a thin otic capsule, absence of the bony modiolus, enlarged nerve canals in the IAC fundus, dysplastic oval and round windows, and an abnormal stapes footplate as well. Sennaroglu ${ }^{13}$ reported that the thin otic capsule may be formed by a thick endosteal layer, and probably the second and third layers are either absent or very thin instead of the usual 3 layers. It may not be possible to observe the normal endosteal layer or differentiate these 3 layers on CT with today's level of radiologic precision. However, we still observed hypodense areas in the region of the fissula ante fenestram in the thin otic capsule in most of our young patients, which are prevalent among healthy children. ${ }^{17}$ This pericochlear hypodensity suggests that the thin otic capsule may have $>1$ layer. With respect to the cystic changes of the vestibule, SCC, and VA, we speculate that they might be caused by the abnormal development of the perilymphatic space.

The disadvantage of this case series is that we did not perform genetic analyses. However, the diagnosis of IP-III can be established with typical imaging features. Radiologists should be familiar to these imaging features, thereby preventing harmful interventions and providing proper genetic counseling.

\section{CONCLUSIONS}

IP-III is a rare genetic inner ear malformation with distinctive imaging features. It involves not only the cochlea and IAC, but also the whole otic capsule, including the vestibule and SCC.

\section{REFERENCES}

1. Nance WE, Setleff R, McLeod A, et al. X-linked mixed deafness with congenital fixation of the stapedial footplate and perilymphatic gusher. Birth Defects Orig Artic Ser 1971;07:64-69 Medline

2. Petersen MB, Wang Q, Willems PJ. Sex-linked deafness. Clin Genet 2008;73:14-23 CrossRef Medline

3. Phelps PD, Reardon W, Pembrey M, et al. X-linked deafness, stapes gushers and a distinctive defect of the inner ear. Neuroradiology 1991;33:326-30 CrossRef Medline

4. Sennaroglu L, Sarac S, Ergin T. Surgical results of cochlear implantation in malformed cochlea. Otol Neurotol 2006;27:615-23 CrossRef Medline

5. Talbot JM, Wilson DF. Computed tomographic diagnosis of $\mathbf{X}$ linked congenital mixed deafness, fixation of the stapedial footplate, and perilymphatic gusher. Am J Otol 1994;15:177-82 Medline

6. Saylisoy S, Incesulu A, Gurbuz MK, et al. Computed tomographic findings of X-linked deafness: a spectrum from child to mother, 
from young to old, from boy to girl, from mixed to sudden hearing loss. J Comput Assist Tomogr 2014;38:20-24 CrossRef Medline

7. Gong WX, Gong RZ, Zhao B. HRCT and MRI findings in X-linked non-syndromic deafness patients with a POU3F4 mutation. Int $J$ Pediatr Otorhinolaryngol 2014;78:1756-62 CrossRef Medline

8. Sennaroğlu L, Bajin MD. Classification and current management of inner ear malformations. Balkan Med J 2017;34:397-411 CrossRef Medline

9. Purcell D, Johnson J, Fischbein N, et al. Establishment of normative cochlear and vestibular measurements to aid in the diagnosis of inner ear malformations. Otolaryngol Head Neck Surg 2003;128:7887 CrossRef Medline

10. Choi JW, Min B, Kim A, et al. De novo large genomic deletions involving POU3F4 in incomplete partition type III inner ear anomaly in East Asian populations and implications for genetic counseling. Otol Neurotol 2015;36:184-90 CrossRef Medline

11. Marlin S, Moizard MP, David A, et al. Phenotype and genotype in females with POU3F4 mutations. Clin Genet 2009;76:558-63 CrossRef Medline
12. Papadaki E, Prassopoulos P, Bizakis J, et al. $\mathrm{X}$-linked deafness with stapes gusher in females. Eur J Radiol 1998;29:71-75 CrossRef Medline

13. Sennaroglu L. Histopathology of inner ear malformations: do we have enough evidence to explain pathophysiology? Cochlear Implants Int 2016;17:3-20 CrossRef Medline

14. Anderson EA, Ozutemiz C, Miller BS, et al. Hypothalamic hamartomas and inner ear diverticula with $\mathrm{X}$-linked stapes gusher syndrome: new associations? Pediatr Radiol 2020;50:142-45 CrossRef Medline

15. Phippard D, Heydemann A, Lechner M, et al. Changes in the subcellular localization of the Brn4 gene product precede mesenchymal remodeling of the otic capsule. Hear Res 1998;120:77-85 CrossRef Medline

16. Phippard D, Lu L, Lee D, et al. Targeted mutagenesis of the POUdomain gene Brn4/Pou3f4 causes developmental defects in the inner ear. J Neurosci 1999;19:5980-89 CrossRef Medline

17. Pekkola J, Pitkaranta A, Jappel A, et al. Localized pericochlear hypoattenuating foci at temporal-bone thin-section $\mathrm{CT}$ in pediatric patients: nonpathologic differential diagnostic entity? Radiology 2004;230:88-92 CrossRef Medline 